\title{
EFFECT OF INTERFACE COUPLING ON THE ENERGY SPECTRUM OF TRIPLE-LAYER FILM. INTERFACE-LOCALIZED MODES
}

\author{
M. Slomian and H. PuszKarski \\ Surface Physics Division, Physics Institute, A. Mickiewicz University, Matejki 48/49 \\ 60-769 Poznań, Poland \\ (Received September 25, 1990)
}

\begin{abstract}
Numerical results of a new (interface-rescaling) theory of normal excitations in a triple-layer film are reported. The film is modelled by three one-dimensional finite chains coupled together; the coupling across the in terface is properly taken into account. The formation process of the film energy spectrum as a result of interface (i.e. inter-chain) coupling is studied, and the exact conditions for the existence of interface-localized modes are established.
\end{abstract}

PACS numbers: 75.70.-i, 73.20.-r, 68.35.-p

\section{Introduction}

Recently, the synthesis and study of layered structures (multilayers, quantum wells, superlattices) have become of great interest. Theoretical studies of such systems have been started only a few years ago (see the review by Dobrzyński [1]). When their physical properties are analysed within a matrix representation, one is faced with the analysis of composite matrices formed out of homogeneous parts corresponding to the individual subsystems, related together through the interface interactions. In general, direct numerical analysis of such large composite matrices involves huge numerical computations. Therefore, alternative analytical approaches are desirable.

The interface rescaling (IR) theory [2] calculates analytically the eigenvalues and eigenvectors of the finite composite system by reducing its eigenproblem to that of one of its individual constituent subsystems. The method, first applied to bilayer films [3], has recently enabled us to propose an exact and complete solution to the eigenvalue problem of a triple-layer film consisting of three (internally 
homogeneous) layers [4]. The IR approach allows us to take proper account of the interface conditions.

In presenting the essentials of our calculations we have chosen a simple model of LCR system, composed of three finite one-dimensional chains (subchains: L, C and $R$ ) separated by interfaces. The aim of this work is to investigate the influence of the interface coupling on the energy spectrum of our composite system and to study the intrinsic properties of the collective modes supported by the superstructure theoretically. The outline of this paper is as follows: in Sec. 2 we start with the presentation of our triple-layer system and its analytical eigensolutions; in. Sec. 3 we study step by step the emergence of collective normal excitations in the system when the interface coupling is made to vary; finally, we discuss the existence conditions for localized states in our system (Sec. 4).

\section{The model}

We consider a system of three identical finite atomic chains (Fig. 1). The boundary chains ( $\mathrm{R}$ - right and $\mathrm{L}$ - left) consist of $N$ atoms, whereas the central (C) chain contains $L-2 N$ atoms. The atoms of the (one-dimensional) system are labelled throughout by $\ell$, ranging from $\ell=0$ to $\ell=L-1$. The energy coupling the chains through the two interfaces is described by the coupling parameter $\varepsilon$. The outer chains $L$ and $R$ are moreover characterized by a surface parameter $A$. In the central chain we distinguish one perturbed atom on either of the two interfaces characterized by the interface parameter $C$. Thus, the system contains six perturbed atoms: The two surface atoms $\ell=0$ and $\ell=L-1$, and the four interface atoms: $\ell=N-1, \ell=N, \ell=L-N-1$ and $\ell=L-N$.

We now write the eigenproblems of each of the chains separately (taking into account intra-chain interactions between nearest neighbours only) in the form of the following three matrix equations:

For the chain L:

$$
\check{A}_{N}^{(\mathrm{L})}(E)\left[\begin{array}{l}
u_{0} \\
u_{1} \\
\vdots \\
u_{N-1}
\end{array}\right]=0 \quad \text { or } \quad \check{A}_{N}^{(\mathrm{L})}(E) \check{u}_{\ell}^{(\mathrm{L})}=0
$$

for the chain $\mathrm{C}$ :

$$
\check{A}_{L-2 N}^{(\mathrm{C})}\left[\begin{array}{l}
u_{N} \\
u_{N+1} \\
\vdots \\
u_{L-N-1}
\end{array}\right]=0 \quad \text { or } \quad \check{A}_{L-2 N}^{(\mathrm{C})}(E) \check{u}_{\ell}^{(\mathrm{C})}=0
$$

for the chain R:

$$
\check{A}_{N}^{(\mathrm{R})}(E)\left[\begin{array}{l}
u_{L-N} \\
u_{L-N+1} \\
\vdots \\
u_{L-1}
\end{array}\right]=0 \quad \text { or } \quad \check{A}_{N}^{(\mathrm{R})}(E) \check{u}_{\ell}^{(\mathrm{R})}=0 .
$$




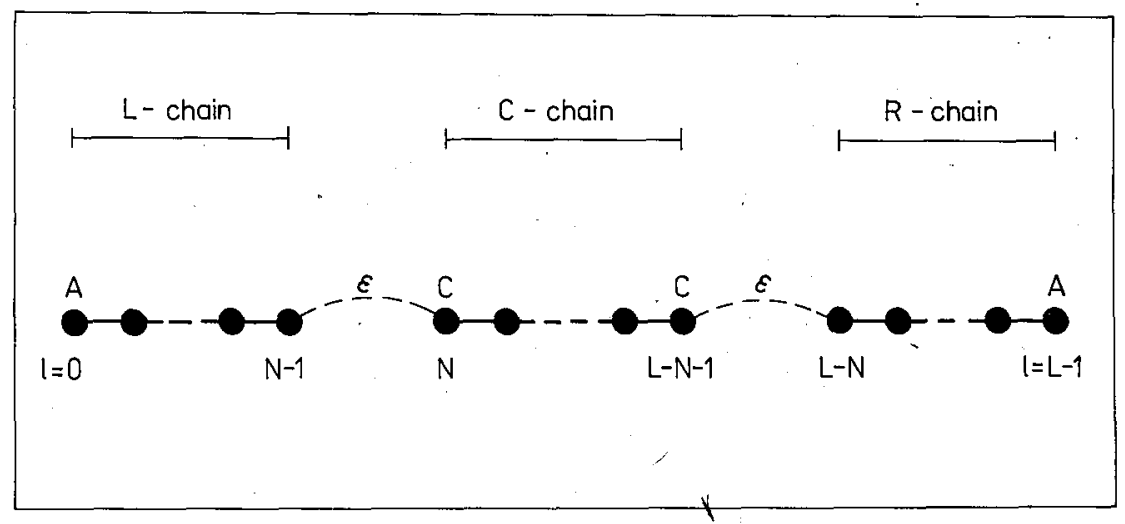

Fig، 1. Model of a system composed of three atomic subchains. The outer subchains $\mathrm{L}$ and $\mathrm{R}$ contain $N$ atoms each, whereas the central subchain $\mathrm{C}$ has $L-2 N$ atoms. The index $\ell$ labels the atoms, from $\ell=0$ to $\ell=L-1$. The "surface" atoms $\ell=0$ and $\ell=L-1$ are disturbed (parameter $A$ ). The two "end" atoms of the central subchain, are also perturbed (parameter $C$ ). Parameter $\varepsilon$ describes the coupling between the four interfacial atoms: $\ell=N-1, \ell=N, \ell=L-N-1$ and $\ell=L-N$.

The upper index (in round parentheses) at the respective matrix $A$ denotes the chain. Likewise, the eigenproblem of the system as a whole (see Fig. 1) is of the matrix form:

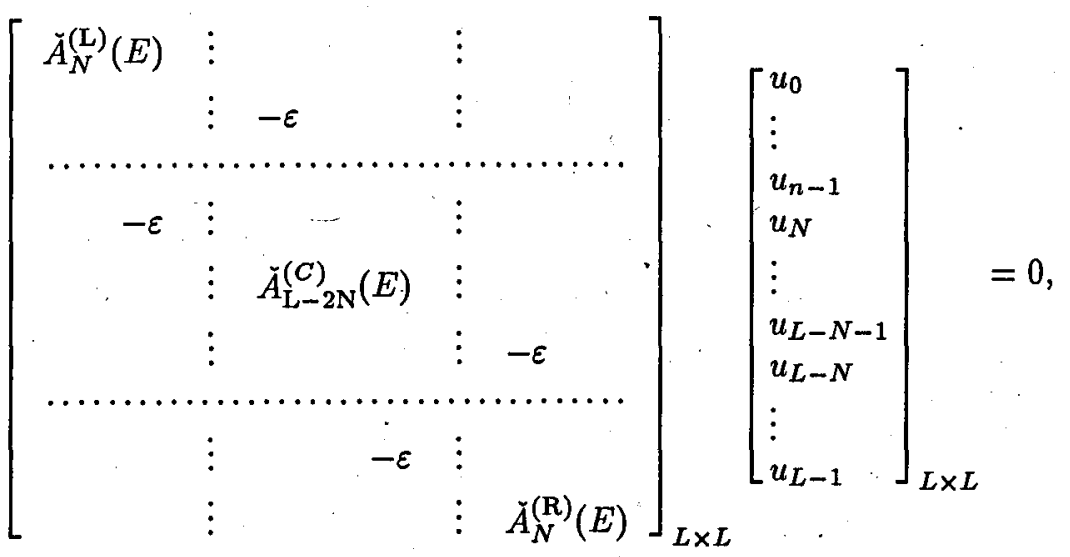

where the submatrices are defined as follows:

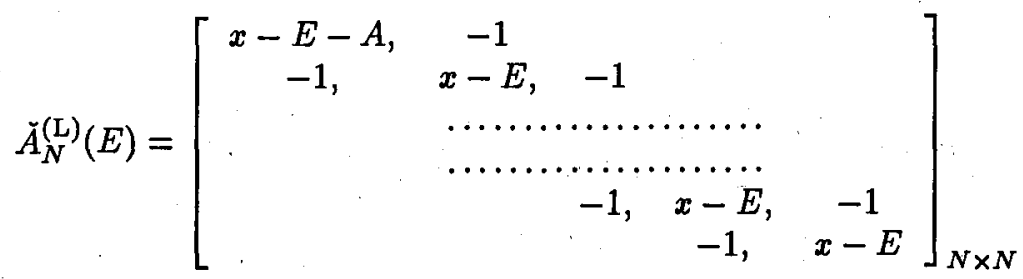




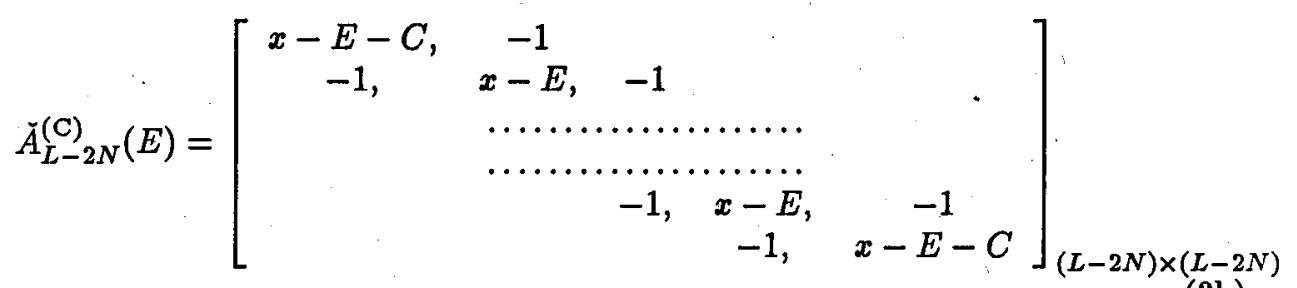

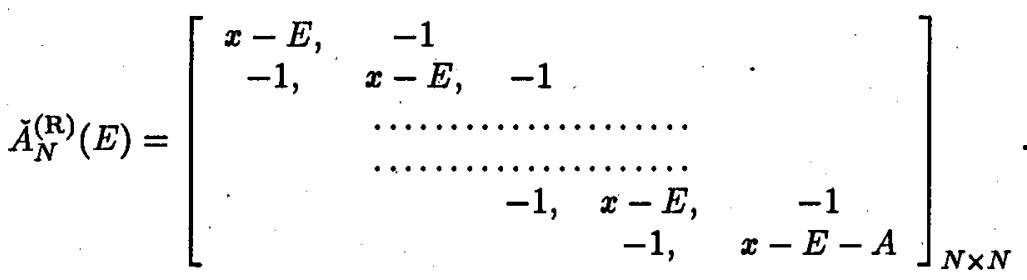

The set of Eqs. (2) can be rewritten in the form of three non-homogeneous sub-sets:

$$
\begin{gathered}
\check{A}_{N}^{(\mathrm{L})}(E)\left[\begin{array}{l}
u_{0} \\
u_{1} \\
\vdots \\
u_{N-1}
\end{array}\right]_{N}=\left[\begin{array}{l}
0 \\
0 \\
\vdots \\
\varepsilon u_{N}
\end{array}\right]_{N}, \\
\check{A}_{L-2 N}^{(\mathrm{C})}(E)\left[\begin{array}{l}
u_{N} \\
u_{N+1} \\
\vdots \\
u_{L-N-1}
\end{array}\right]_{L-2 N}=\left[\begin{array}{l}
\varepsilon u_{N-1} \\
0 \\
\vdots \\
\varepsilon u_{L-N}
\end{array}\right]_{L-2 N}, \\
\check{A}_{N}^{(\mathrm{R})}(E)\left[\begin{array}{l}
u_{L-N} \\
u_{L-N+1} \\
\vdots \\
u_{L-1}
\end{array}\right]_{N}=\left[\begin{array}{l}
\varepsilon u_{L-N-1} \\
0 \\
\vdots \\
0
\end{array}\right]_{N} .
\end{gathered}
$$

(Obviously, while putting $\varepsilon=0$ everywhere we would obtain in place of (2) or (4) three eigenproblems of the type (1) for the three "subchains" separately.)

Since our model is endowed with symmetry (see Fig. 1), we expect to arrive at two kinds of solutions: symmetric and antisymmetric. On re-scaling the interface conditions by the method proposed in [2] we obtain characteristic equations of the following form [4]:

$$
\begin{aligned}
& \frac{\cos \left(\frac{L+1}{2}-N\right) \tau}{\cos \left(\frac{L-1}{2}-N\right) \tau}=C+\varepsilon^{2} \frac{\sin N \tau-A \sin (N-1) \tau}{\sin (N+1) \tau-A \sin N \tau} \\
& \frac{\sin \left(\frac{L+1}{2}-N\right) \tau}{\sin \left(\frac{L-1}{2}-N\right) \tau}=C+\varepsilon^{2} \frac{\sin N \tau-A \sin (N-1) \tau}{\sin (N+1) \tau-A \sin N \tau}
\end{aligned}
$$

the former (5a) corresponding to symmetric and the latter (5b) to antisymmetric solutions. We write the dispersion relation as follows:

$$
E-x=-2 \cos \tau \text {. }
$$


Having recourse to Ref. [2], we obtain the eigenfunctions in the following form [4]:

$$
\begin{gathered}
u_{\ell}=\varepsilon \frac{\sin (\ell+1) \tau-A \sin \ell \tau}{\sin (N+1) \tau-A \sin N \tau} \quad \text { for } \ell=0,1, \ldots, N-1 ; \\
u_{\ell}=\left\{\begin{array}{lc}
\frac{\sin \left(\frac{L-1}{2}-\ell\right) \tau}{\sin \left(\frac{L-1}{2}-N\right) \tau} & \text { (antisymmetric modes) } \\
\frac{\cos \left(\frac{L-1}{2}-\ell\right) \tau}{\cos \left(\frac{L-1}{2}-N\right) \tau} & \text { for } \ell=N, N+1, \ldots, L-N-1 ;
\end{array}\right. \\
u_{\ell}=\mp u_{L-1-\ell} \text { for } \ell=L-N, L-N+1, \ldots, L-1 .
\end{gathered}
$$

The roots of the characteristic equations (5) can be real (corresponding to energy levels lying within the permitted band) or complex. The complex solutions can be of two types: with energy values lying below the lower edge of the band (LB - lying below; $\tau=\mathrm{i} t$ ), or with energy values lying above its upper edge (LA - lying above; $\tau=\pi+i t)$. We refer to solutions with complex wave vector $\tau$ and energy lying outside the permitted band as localized solutions. The amplitudes of localized modes decrease exponentially as one moves away from the site on which the mode is localized. We obtain the equations for the complex part of the wave vector (for solutions lying below the band) putting $\tau=i t$ into the characteristic equations $(5 \mathrm{a}, 5 \mathrm{~b})$, in the following form:

$$
\begin{aligned}
& \frac{\cosh \left(\frac{L+1}{2}-N\right) t}{\cosh \left(\frac{L-1}{2}-N\right) t}=C+\varepsilon^{2} \frac{\sinh N t-A \sinh (N-1) t}{\sinh (N+1) t-A \sinh N t} \\
& \frac{\sinh \left(\frac{L+1}{2}-N\right) t}{\sinh \left(\frac{L-1}{2}-N\right) t}=C+\varepsilon^{2} \frac{\sinh N t-A \sinh (N-1) t}{\sinh (N+1) t-A \sinh N t}
\end{aligned}
$$

where (8a) corresponds to symmetric solutions and (8b) to antisymmetric solutions. Similarly, we get the eigenfunctions $u_{\ell}(\tau)$ :

$$
\begin{gathered}
u_{\ell}=\varepsilon \frac{\sinh (\ell+1) t-A \sinh \ell t}{\sinh (N+1) t-A \sinh N t} \quad \text { for } \ell=0,1, \ldots, N-1 ; \\
u_{\ell}=\left\{\begin{array}{cc}
\frac{\sinh \left(\frac{L-1}{2}-\ell\right) t}{\sinh \left(\frac{L-1}{2}-N\right) t} \quad \text { (antisymmetric modes) } \\
\frac{\cosh \left(\frac{L-1}{2}-\ell\right) t}{\cosh \left(\frac{L-1}{2}-N\right) t} & \text { for } \ell=N, N+1, \ldots, L-N-1 ;
\end{array}\right. \\
u_{\ell}=\mp u_{L-1-\ell} \quad \text { for } \ell=L-N, L-N+1, \ldots, L-1 .
\end{gathered}
$$

In this case, the energy is given by:'

$$
E-x=-2 \cosh t .
$$


For modes with the energy values lying above the band, putting $\tau=\pi+\mathrm{i} t$, we get the following characteristic equations:

$$
\begin{aligned}
& -\frac{\cosh \left(\frac{L+1}{2}-N\right) t}{\cosh \left(\frac{L-1}{2}-N\right) t}=C-\varepsilon^{2} \frac{\sinh N t+A \sinh (N-1) t}{\sinh (N+1) t+A \sinh N t} \\
& -\frac{\sinh \left(\frac{L+1}{2}-N\right) t}{\sinh \left(\frac{L-1}{2}-N\right) t}=C-\varepsilon^{2} \frac{\sinh N t+A \sinh (N-1) t}{\sinh (N+1) t+A \sinh N t}
\end{aligned}
$$

as well as the dispersion relation:

$$
E-x=2 \cosh t
$$

and the eigenfunctions:

$$
\begin{gathered}
u_{\ell}=(-1)^{(\ell+N)} \varepsilon \frac{\sinh (\ell+1) t-A \sinh \ell t}{\sinh (N+1) t-A \sinh N t} \quad \text { for } \ell=0,1, \ldots, N-1 ; \\
u_{\ell}=(-1)^{(\ell-N)}\left\{\begin{array}{lc}
\frac{\sinh \left(\frac{L-1}{2}-\ell\right) t}{\sinh \left(\frac{L-1}{2}-N\right) t} & \text { (antisymmetric modes) } \\
\frac{\cosh \left(\frac{L-1}{2}-\ell\right) t}{\cosh \left(\frac{L-1}{2}-N\right) t} & \text { for } \ell=N, N+1, \ldots, L-N-1 ;
\end{array}\right. \\
u_{\ell}=\mp u_{L-1-\ell} \text { for } \ell=L-N, L-N+1, \ldots, L-1 .
\end{gathered}
$$

Equations (11a) and (11b) correspond to the symmetric and antisymmetric solutions, respectively.

Owing to the existence of localized modes it is necessary that the parameters $A, C, \varepsilon$ and $N$ assume appropriate values. The problem will be discussed in detail further on. Here however,.it should by stated that whereas the influence of the surface parameter $A[5,6]$ and the interface parameter $C[7]$ on the energy spectrum has been the subject of studies, the introduction of the interface coupling $\varepsilon$ is a novel feature of our present paper. Our approach enables us to study the spectrum formation of the system as a whole by evolving the respective spectra of the three subsystems under variations of the interface coupling $\varepsilon$.

\section{The emergence of collective properties of the spectrum}

We shall now follow the process of the spectrum formation of our composite system as a result of inter-chain coupling (we assume for simplicity $C=0$ and $A=0$ ). Either subchain $\mathrm{L}$ and $\mathrm{R}$ consists of 3 atoms, whereas the subchain $\mathrm{C}$ comprises 5 atoms. As long as the subchains exist apart $(\varepsilon=0)$ the spectrum looks like that shown in Figs. 2(b)-(d). Let us denote the energy levels by two indices: one for the subchain and one for the mode (starting from the one with the lowest energy). The composition of the three spectra leads to the spectrum of Fig. 2(a). 


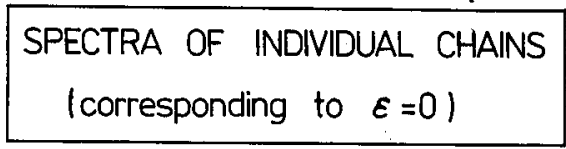

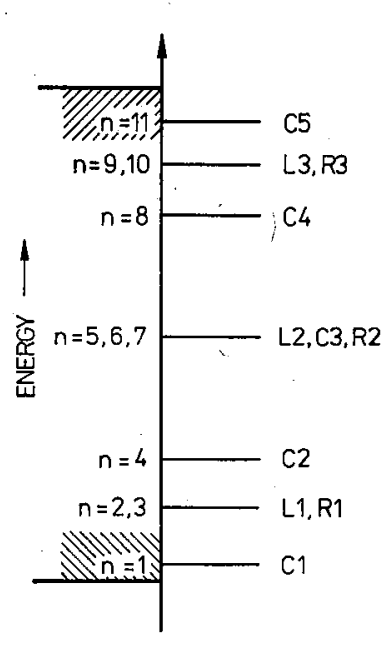

a)
L-Chain

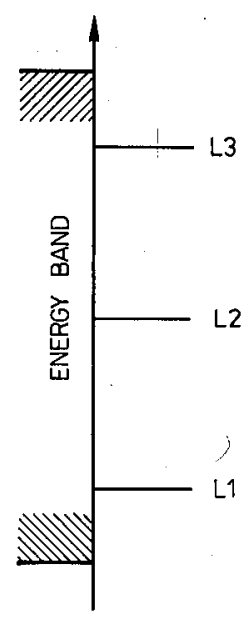

b)

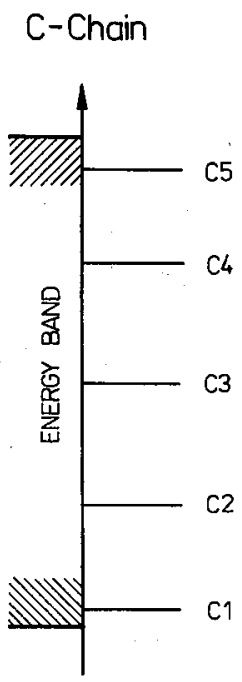

c)

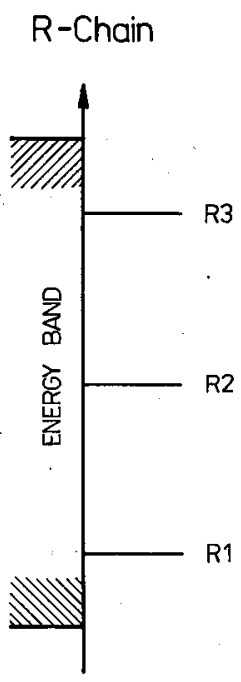

d)

Fig. 2. The spectra of uncoupled chains $L, C$ and $R$. The system consists of a central subchain comprising 5 atoms and of outer subchains comprising 3 atoms each. The parameters $A$ and $C$ are zero; coupling parameter $\varepsilon=0$. (a) shows the spectrum arisen by superposition of the spectra (b), (c) and (d) for $\varepsilon=0$.

The modes originating in the subchains $L$ and $R$ are obviously two-fold degenerate (the two subchains are identical). However, the mode existing in the centre of each of the component spectra becomes three-fold degenerate on composition of the three spectra. In Fig. 2(a) we have denoted "collectivized" modes by an index labelling the modes of the system as a whole (the mode $\mathrm{L} 1$ is the mode with the lowest energy in the chain $\mathrm{L}$; the mode R1 is the one with the lowest energy in the chain $\mathrm{R}$; the modes are now denoted as $n=2, n=3$ ). In Fig. 3 we are able to follow how coupling $\varepsilon \neq 0$ removes the degeneracy. For $\varepsilon=1$ ("bulk" coupling) the spectrum is that of a monoatomic chain; a further increase in coupling "shifts" the two lowest lying and the two highest lying modes out of the band.

The effectt of $\varepsilon$ on the mode profiles is also of interest. We shall give especial attention to the profiles of the three lowest lying modes (Fig. 4). At $\varepsilon \approx 0$, Fig. 4(a), the amplitude of the L-modes differs from zero only in the left chain (the R-modes and C-modes behave likewise). As $\varepsilon$ becomes non-zero, the modes begin to "feel" their presence mutually. It then becomes meaningful to speak of the emergence of 


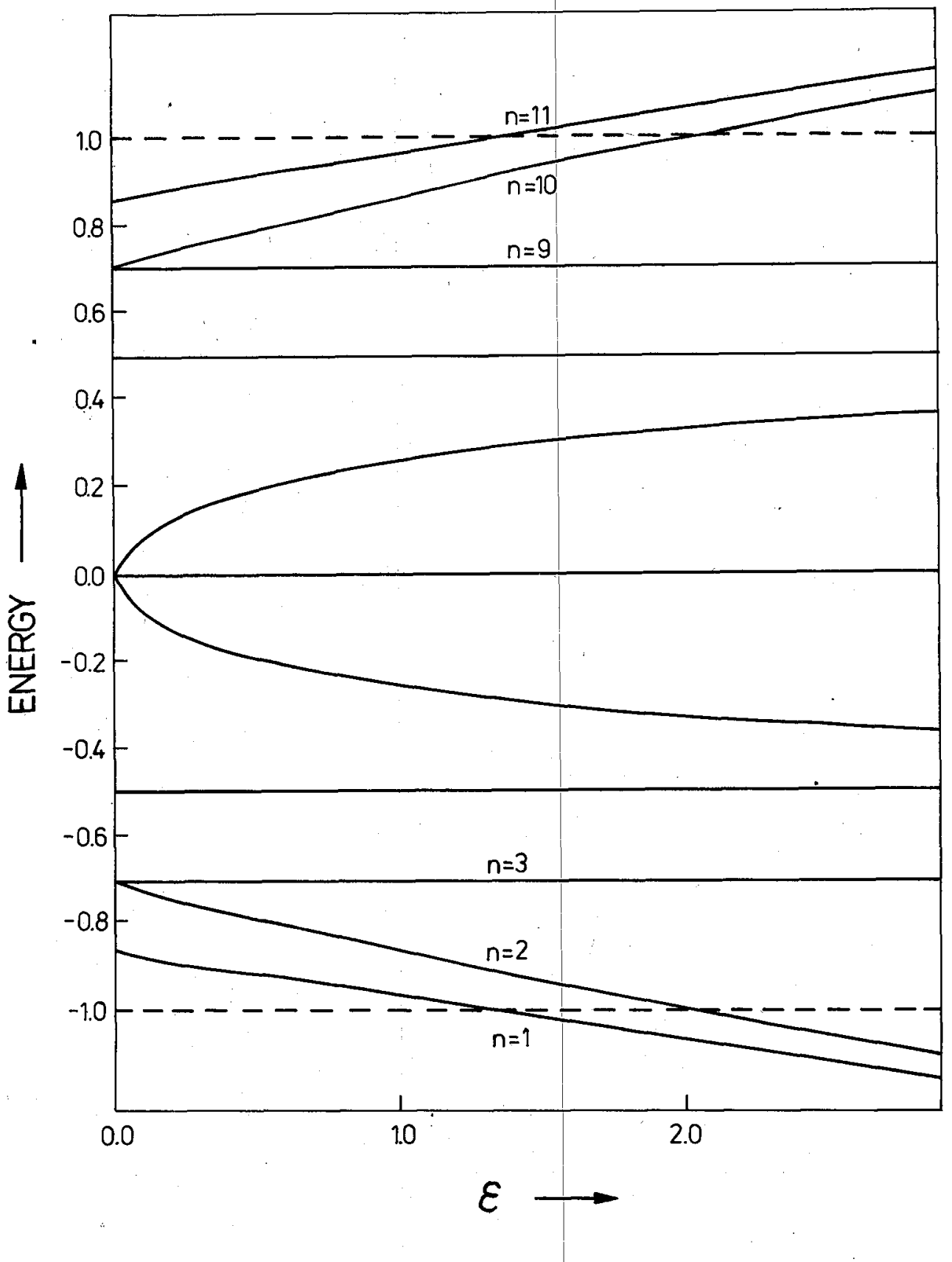

Fig. 3. Influence of the coupling parameter $\varepsilon \neq 0$ on the energy spectrum shown in Fig. 2. Coupling removes the initial degeneracy (see, e.g. the modes $n=2$ and $n=3$ ) and causes four modes to "move out" of the band (its edges are dashed). 

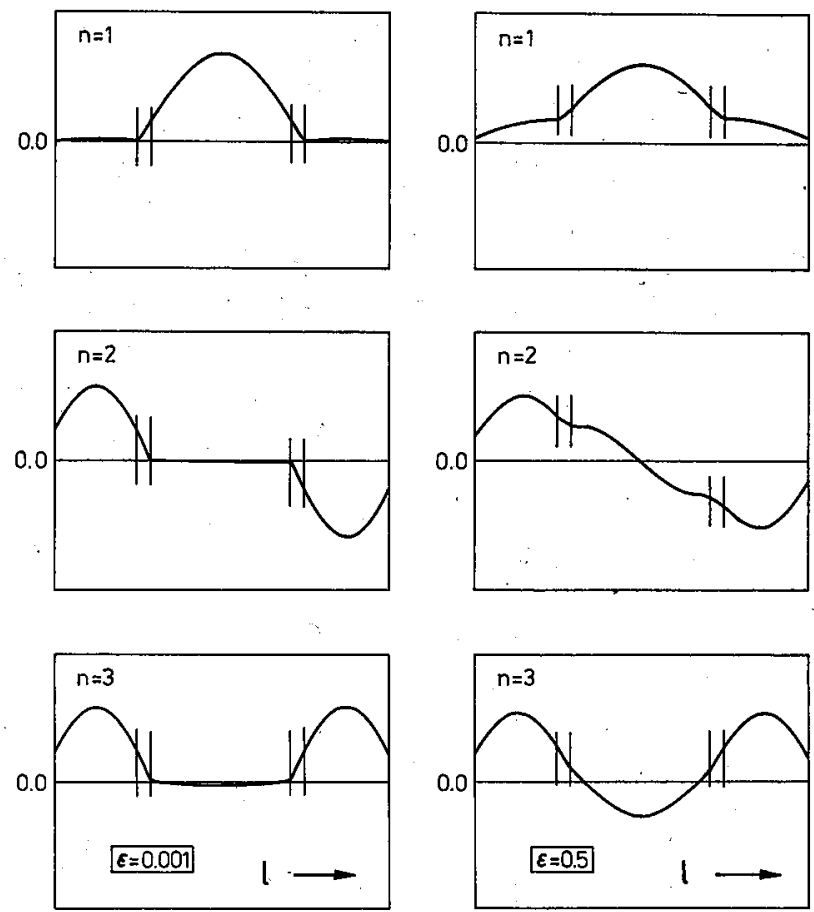

(b)
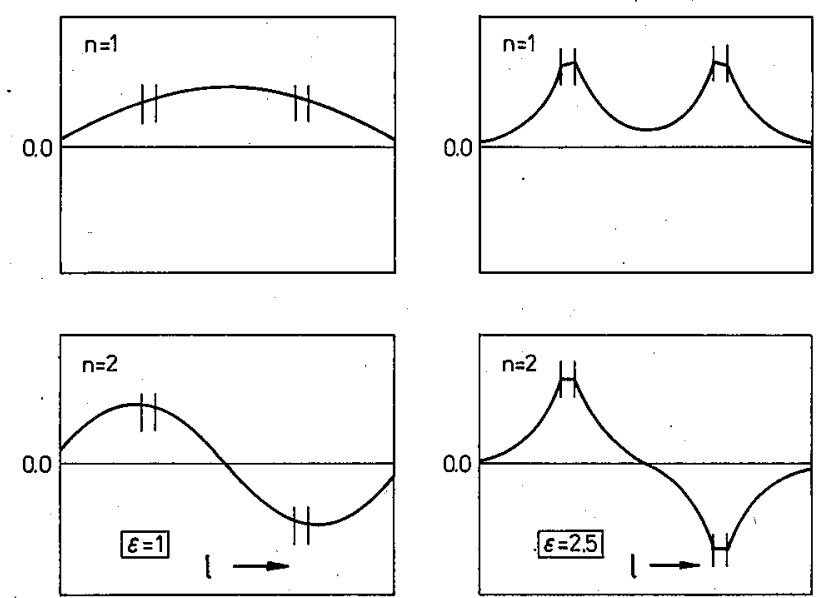

Fig. 4. The change in profiles under the effect of coupling $\varepsilon \neq 0$ for the lowest modes of the composite system (the respective numbers of atoms are: $L=25, N=7$; perturbation parameters: $A=C=0$ ). (a) The modes are: one with the lowest energy is of the type $\mathrm{C}$, two higher modes are of the type LR. At weak coupling $\varepsilon$ non-zero amplitudes occur where the mode is located, growing $\varepsilon$ leads to collectivization of the modes. (b) For $\varepsilon=1$ the profiles traverse the interface smoothly; at great $\varepsilon$, the modes become localized. 
LR-type modes (originating in the outer chains) and a C-type mode originating in the central chain; for very small $\varepsilon$, their amplitudes differ significantly from zero only in the region (or regions) of their origin. We refer to such a mode as "located" in that region. Thus, e.g. the mode $n=1$ stems from the central chain and, consequently, is "located" in the chain C; great amplitudes arise on this chain only. An increase in $\varepsilon$ causes the amplitudes to become "diffluent" over the whole system, and at $\varepsilon=1$ the profile traverses the interface "smoothly" (see Fig. 4(b)). For $\varepsilon=2.5$ one gets a localized mode, the energy of which lies below the lower edge of the band; the amplitude of its profile is maximal on the interface (see Fig. 4(b)) and decreases exponentially with the distance from the latter. The two modes $n=1$ and $n=2$ are apt to become localized (by the way, they differ in symmetry, see Sec. 2).

Henceforth, we shall be distinguishing location and localization. Whereas location will refer to the chain that is occupied by a given mode, localization will refer to the perturbed atom on which the amplitude of the mode is maximal.

Since the mode with the lowest energy is always symmetric, two out of the three lowest modes of the composite system have to be symmetric and one antisymmetric. Hence, when the chains become coupled, one of the symmetric modes has to give rise to an antisymmetric mode: the mode $n=2$, which was symmetric in $\mathrm{L}$ and $\mathrm{R}$, has to be antisymmetric when being a mode of the system as a whole (see Fig. 4(a)). With increasing $\varepsilon$ the modes become more and more strongly "shared" by the system as a whole, i.e. they become collectivized. The concept of location introduced above renders adequately the nature of the mode at small $\varepsilon$, in that it points to the sub-system in which the mode originates. The concepts of location and collectivization are mutually reciprocal: the more strongly a mode becomes collectivized, the more weakly it is located.

We now proceed to consider an interesting system of three chains containing the same number of atoms (e.g. $L=3 \times 7$; Fig. 5). At first, all the levels are three-fold degenerate (Fig. 5(a)); moreover, at zero coupling, all three (degenerate) modes have the same symmetry. Now consider the modes $n=1, n=2$ and $n=3$. When coupling is "switched on", two modes of the type LR form (as preoviously) a symmetric-antisymmetric pair of modes, whereas the third (C-type) mode remains symmetric. Already at very low $\varepsilon$, two of the three modes, the two symmetric ones, still having closely lying energy values collectivize very rapidly. Referring to the considerations of [7] regarding hybridization, one arrives at the conclusion that at $\varepsilon \approx 0$ the modes $n=1$ and $n=3$ are in a condition of "pseudohybridization" (since they have identical symmetries and almost equal energy values). The third mode of the triplet, being antisymmetric, differs in symmetry from the other two and thus does not undergo "pseudohybridization". (In the next triplet: $n=4$, $n=5$ and $n=6$, inversely, collectivization due to pseudohybridization "attacks" the antisymmetric modes).

The quantitative distribution of atoms among the (central and outer) subchains affects the spectrum of the system profoundly. First let us consider what happens at the edges of the band. In Fig. 6(a), the lowest mode came from the chain $C$ whereas the next two were of the type LR. In Fig. 5(a) we dealt with a different situation: two LR-modes and one C-mode possessed equal energy values. 
(a)

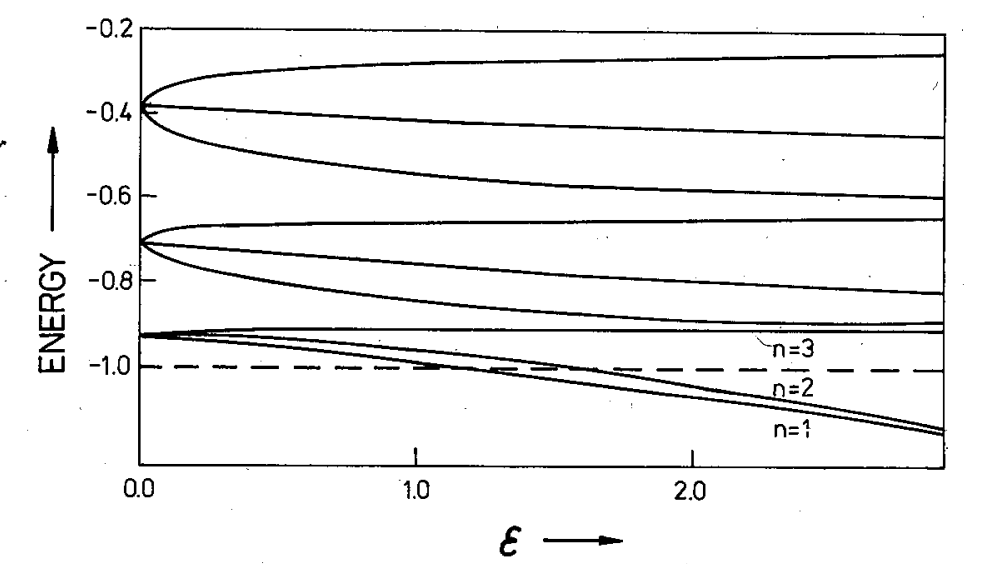

(b)
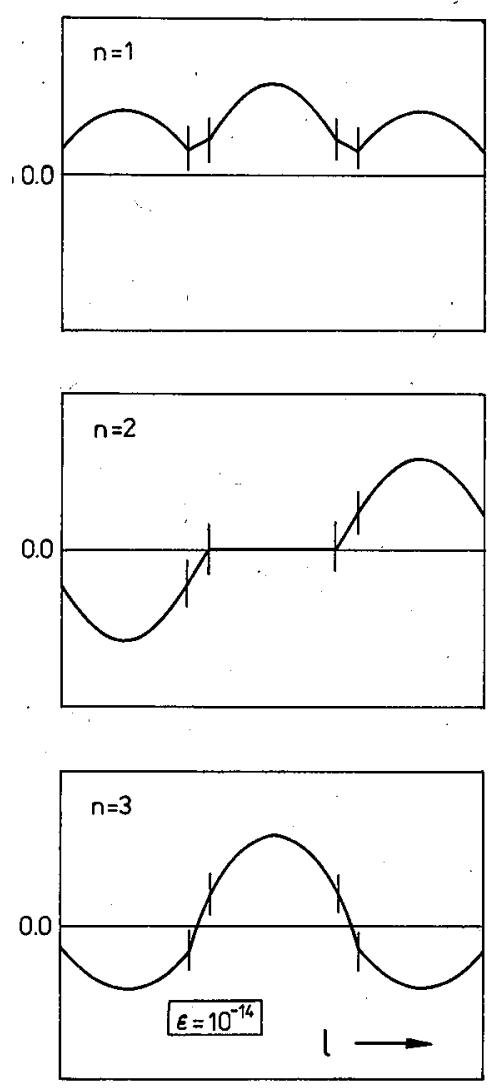

Fig. 5. System with three-fold degenerate levels at $\varepsilon=0(L=21, N=7, A=C=0)$. (a) Interface coupling $\varepsilon \neq 0$ removes the degeneracy. (b) Symmetry features of the three lowest modes. 
(a)

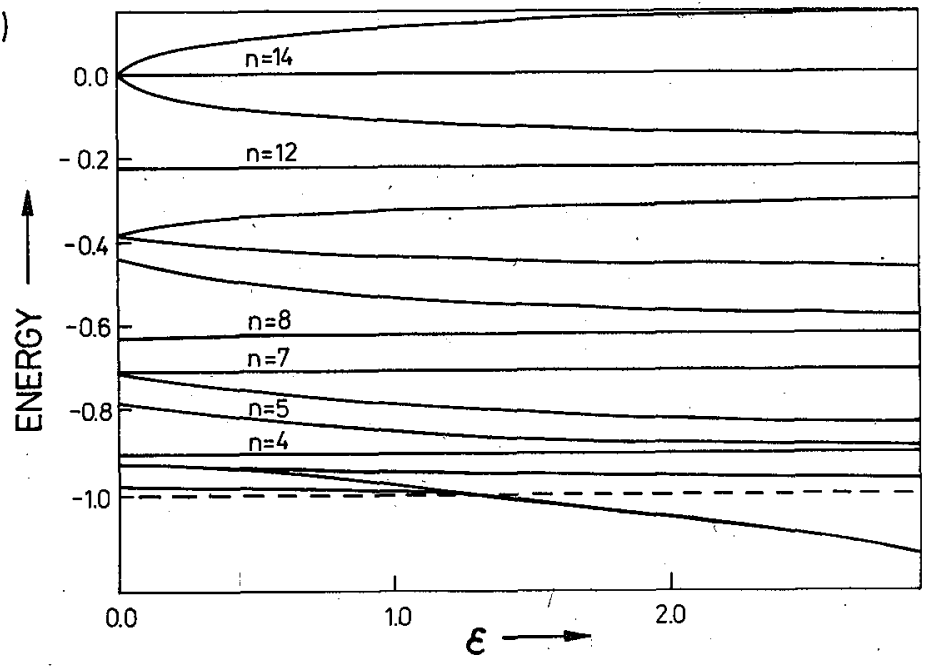

(b)
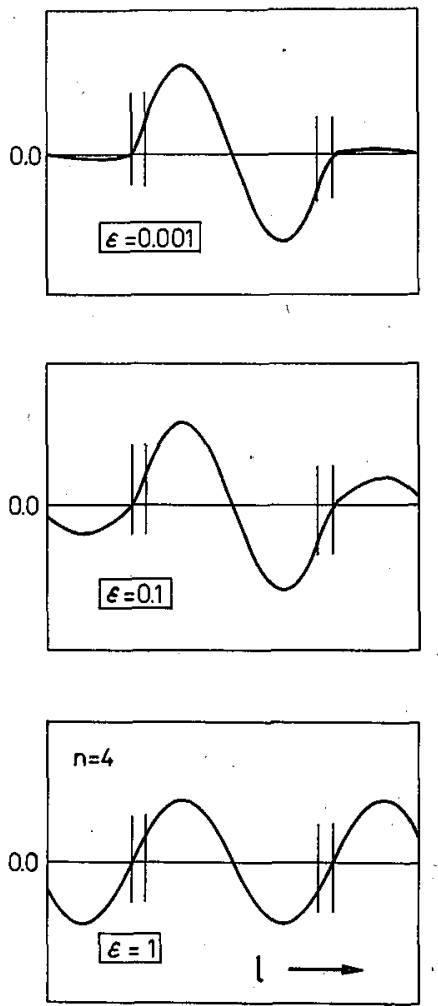

Fig. 6. (a) Spectrum ( $L=25, N=9, A=C=0$ ) exhibiting the modes with energy independent of $\varepsilon(n=4,7,8,12,14)$. (b) Example of the mode $n=4$ which undergoes collectivization, one of the interface atoms remaining immobile throughout. 
Since localization always bears on the two modes with the lowest (and/or the two with the highest) energy, quantitatively different distributions over the subsystems must necessarily involve localization of differently located modes. Moreover, knowledge of the location of a given mode is essential in determining the influence of the perturbation parameters $A$ and $C$ on its prospective localization.

It is noteworthy that, under certain conditions, modes can occur with an energy independent of the coupling parameter $\varepsilon$. Figure 6(a) shows an entire set of such modes $(n=4,7,8,12,14)$. Now let us have a look (Fig. 6(b)) at the mode labelled $n=4$, which is located on the central (C) chain. It turns out that the key to the interpretation of the constant energy lies with the amplitude on the interfacial atoms: if the mode is located on the chain $\mathrm{C}$ with zero amplitude on an interfacial atom (an atom belonging to an outer chain) its energy value does not vary under the effect of coupling; inversely, if a mode is located on the outer chains with zero amplitude on an interfacial atom (in the chain C!) its energy value too will not be sensitive to coupling. Quite generally, the following rule holds: if a mode exhibits a node on an interface when $\varepsilon=0$, it will retain its energy value constant throughout the whole range of variability of $\varepsilon \neq 0$.
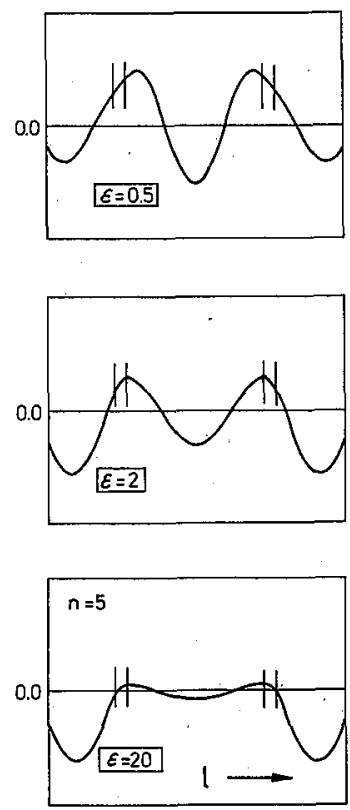

Fig. 7. Example of the mode ( $n=5)$, whose energy is $\varepsilon$-dependent; with growing $\varepsilon$, the mode reduces one of its amplitudes on the interface to zero. (Parameters are the same as in Fig. 6).

The behaviour of modes, which at $\varepsilon=0$ exhibit no node on interfacial atoms, is quite different: initially, their energy is strongly dependent on growing $\varepsilon$, and 
subsequently stabilizes as $\varepsilon \rightarrow \infty$ (see e.g. Fig. 6(a)). The latter property results from the fact that strong coupling $\varepsilon$ always reduces one of the amplitudes on the interface to zero (see Fig. 7); this, in fact, is the reason why the energy of these modes becomes constant, as it did in the case of the modes discussed above.

\section{The conditions for the existence of localized modes}

Taking into account the limits of the formulas $(8 \mathrm{a}, \mathrm{b})$ and $(11 \mathrm{a}, \mathrm{b})$ for $t \rightarrow 0$ we obtain the conditions for the existence of out-of-band localized modes (see, e.g. [8]). For under-band ("localized below" - LB) modes we get:

$$
\begin{gathered}
\varepsilon=(1-C) \frac{N+1-A N}{N+A-A N} \quad \text { for symmetric modes, } \\
\varepsilon=\left(\frac{L-2 N+1}{L-2 N-1}-C\right) \frac{N+1-A N}{N+A-A N} \quad \text { for antisymmetric modes. }
\end{gathered}
$$

Whereas for over-band ("localized above" - LA) modes we get:

$$
\begin{gathered}
\varepsilon=(1+C) \frac{N+1+A N}{N-A+A N} \quad \text { for symmetric modes, } \\
\varepsilon=\left(\frac{L-2 N+1}{L-2 N-1}+C\right) \frac{N+1+A N}{N-A+A N} \quad \text { for antisymmetric modes. }
\end{gathered}
$$

For the particular case considered in the previous section $(A=0, C=0)$ the existence conditions for LB- and LA-modes assume the following forms:

$$
\begin{gathered}
\varepsilon=\frac{N+1}{N} \quad \text { for symmetric modes, } \\
\varepsilon=\frac{L-2 N+1}{L-2 N-1} \frac{N+1}{N} \quad \text { for antisymmetric modes. }
\end{gathered}
$$

Figure 8 shows $\varepsilon$ vs. $N$ as given by Eqs. (14e,f). This figure shows regions denoted by two numbers, the one giving the number of modes localized below the band and the other giving the number of modes localized above it. The region $(0,0)$ contains no localized modes. In the region $(1,1)$ we have one mode below the band (one LB-mode) and one mode above the band (one LA-mode), both of them symmetric. In the region $(2,2)$ there exist two modes below the band and two above the band (differing in symmetry).

Let us now consider, $\varepsilon$ being constant, how the energy of the modes lying in the lower part of the band varies with varying $N$. Figure 9 visualizes the changes in energy of the four lowest modes; two of them are localized and two are bulk. The localized mode with the lower energy is symmetric whereas the one with the higher energy is antisymmetric. The localization is the strongest for $N$ between 5 and 7 , i.e. if the number of atoms in the chain $\mathrm{C}$ is close to the number of atoms in either of the outer chains. The two interfaces are then equidistant from the boundaries (the surface) of the system. The symmetric and antisymmetric modes behave 


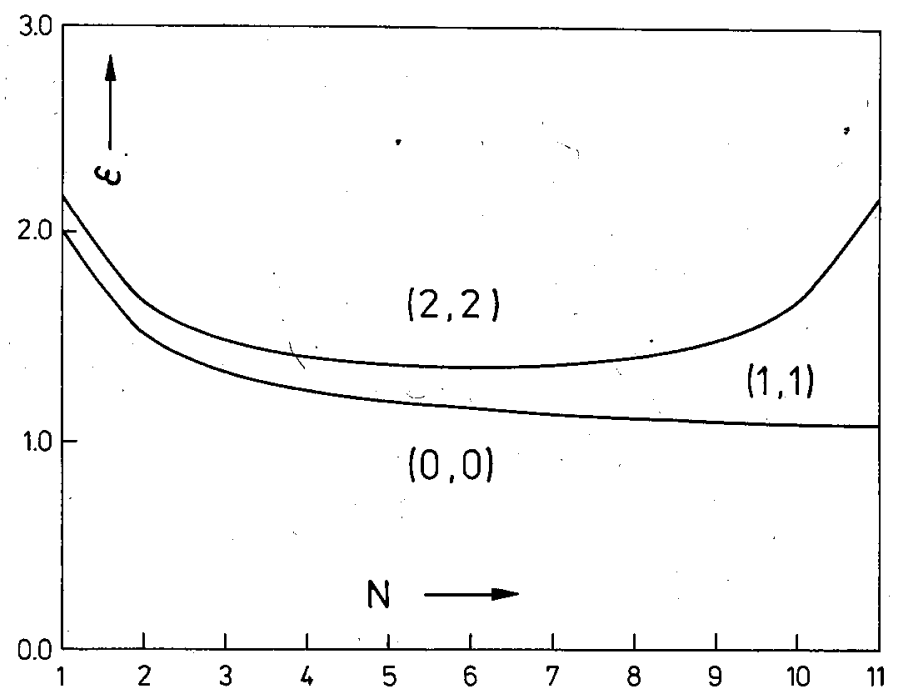

Fig. 8. The existence conditions for localized modes in the $N-\varepsilon$ plane $(L=25$, $A=C=0$ ). Notations: $(0.0)-$ region with no localized modes, $(1,1)-$ region with one LA-mode and one LB-mode, $(2,2)$ - region with two LA-modes and two LB-modes.

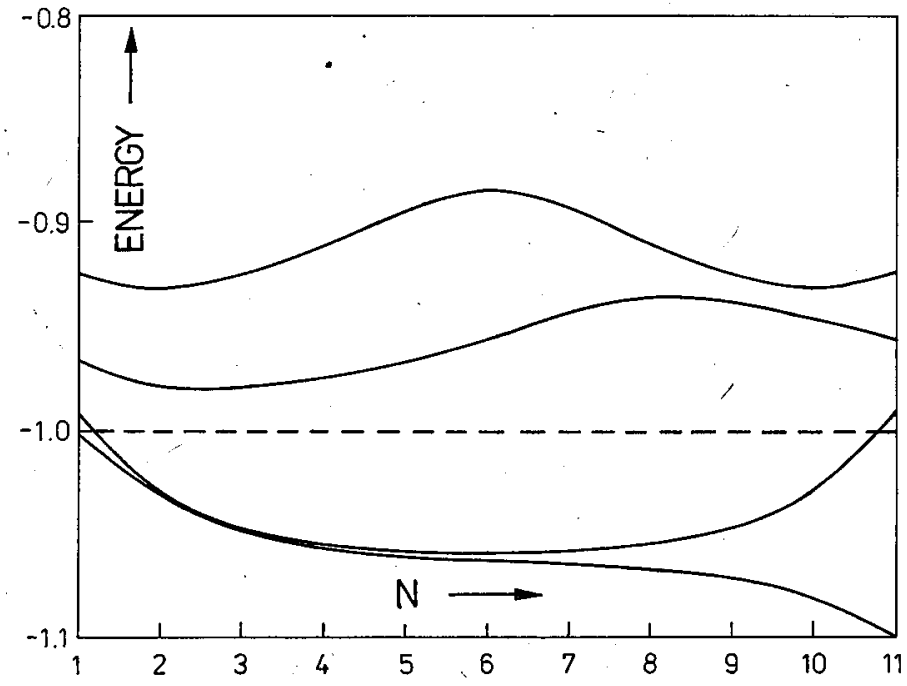

Fig. 9. The energy of the four lowest lying modes vs. $N(L=25, \varepsilon=1.5, A=C=0)$. The dashed line is the lower edge of the band. The two lowest modes move out of the band for certain values of $N$ (they become localized). 

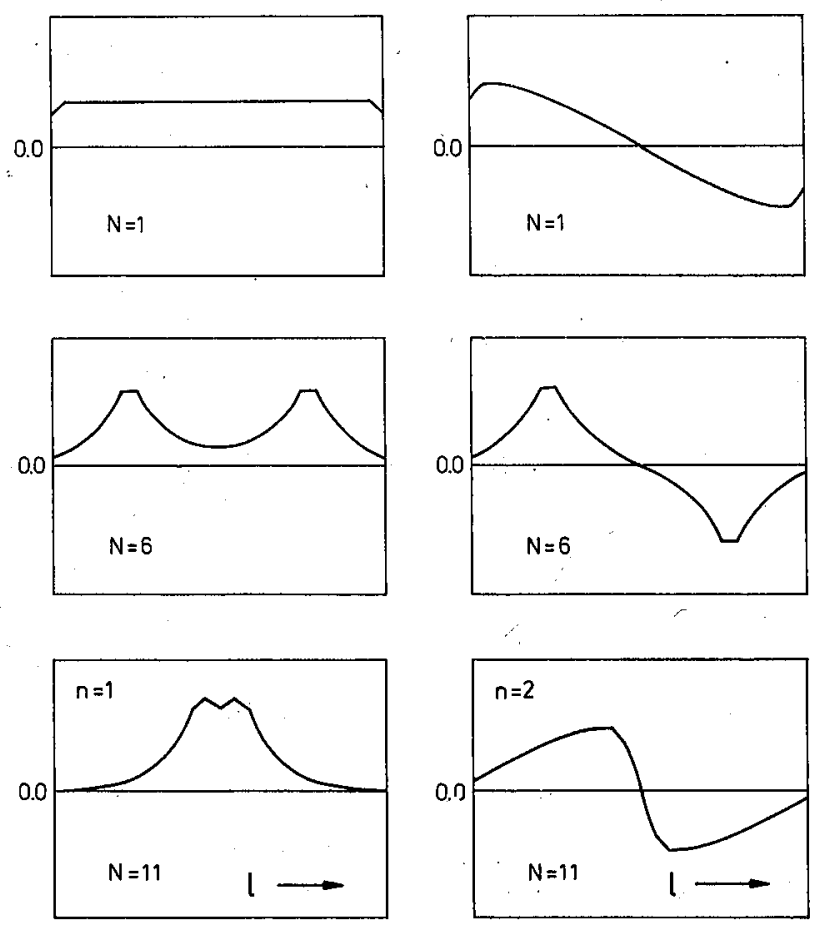

Fig. 10. The mode profiles (for different $N$ ) showing how localization wanders with the interface $(L=25, \varepsilon=2, A=C=0)$.

differently with growing $N$; whereas the symmetric mode enhances its localization, the antisymmetric mode delocalizes (i.e. it "returns" to the band). Figure 10 shows the respective profiles of the two localized modes in their dependence on $N$.

\section{References}

[1] L. Dobrzyński, Surf. Sci. Rep. 6, 119 (1986).

[2] H. Puszkarski, Acta Phys. Pol. A74, 701 (1988).

[3] H. Puszkarski, Solid State Commun. 72, 887 (1989).

[4] H. Puszkarski, Acta Phys. Pol. A78, 753(1990).

[5] H. Puszkarski, Acta Phys. Pol. 36, 675 (1969).

[6] H. Puszkarski, Surf. Sci. 34, 125 (1973).

[7] H. Puszkarski, Prog. Surf. Sci. 25, 155 (1987).

[8] E.N. Economou, Green's Function in Quantum Physics, 2nd ed. Springer-Verlag, Berlin 1983. 